\title{
Gross anatomy for physical therapy course
}

\author{
Ward M. Glasoe ${ }^{1 *}$, James R. Carey, Brendan J. Dougherty ${ }^{1}$ \\ 'Division in Physical Therapy, Department of Rehabilitation Medicine, University of Minnesota
}

\section{Dear Editor,}

The study of anatomy supported with human dissection is foundational to training of health science professionals. For a student, cadaver dissection offers an active learning experience. For the training of physical therapists, we created a manual that efficiently instructs the dissection of the whole body over the course of 30 laboratory periods. In the spirit of academic collegiality, this letter provides health science educators a direct download link (https://hdl.handle. net/11299/218174) to the dissection manual hosted by the University of Minnesota (USA) Digital Conservancy. No registration is required, the download is free, and the PDF file of the dissection manual can be reproduced or adapted for any educational use.

The Journal of Health Sciences has international scope that reaches a vast audience of health professionals. Its readership includes health science educators, with human anatomy serving as the pedagogical foundation of curricular training. In higher education and medical universities, active learning laboratories involve the dissection of human cadavers. For this purpose, we share a download of the 31-page manual that is free to reproduce or adapt for any educational use.

The manual is authored by three Ph.D. trained physical therapists, each having distinctly different clinical training and academic research backgrounds. Ward M. Glasoe was trained in orthopedics, James R. Carey in neurology, and Brendan J. Dougherty in respiratory neurophysiology. Their professional careers intersect in teaching the Gross Anatomy for Physical Therapy course at the University of Minnesota. Anatomy is the first course in the Doctor of Physical Therapy educational curriculum. Prior to enrollment, the student's complete undergraduate anatomy. The Anatomy Bequest Program at the University of Minnesota

${ }^{*}$ Corresponding author: Ward M. Glasoe, Division in Physical Therapy, Department of Rehabilitation Medicine, University of Minnesota, 500

Boynton Health Service Bridge, 410 Church St. SE, Minneapolis, MN 55455, USA e-mail: glaso008@umn.edu

Submitted: 04. February 2021/Accepted: 05. February 2021

DOI: https://doi.org/10.17532/jhsci.2021.1246 supports the course by providing the cadavers and dissection classroom laboratories. The laboratories are ventilated to meet standards of regulating agencies, equipped with dissection tables and tools, and an assortment of anatomical skeletons and models. First aid kits and eyewash stations are available in the laboratory if needed. Locker rooms are nearby to allow students and faculty to change into medical scrubs that are worn during dissection.

The anatomy dissection manual guides the students through a systematic process of whole body dissection taught over $30(2 \mathrm{~h})$ laboratory periods. The manual primarily instructs dissection of the musculoskeletal system along with the peripheral nerves, arteries, and heart. Abdominal and pelvic viscera are studied briefly and then removed to allow access to neuromusculoskeletal structures located inside the body cavities. The brain is removed and studied as a prelude to a more intensive neuroscience course offered later in the curriculum. This instruction of structure serves as the basis for training human movement science. Use of the manual in academics should be augmented with other published materials. Our students frequently refer to one or more anatomy atlases provided in the laboratory and may also bring other course materials with them to address the higher learning objectives for the class.

Although other dissection manuals are available in the marketplace, those dissection guidelines teach an in-depth, multisystem approach that targets the training of anatomists or physicians. In contrast, this dissection manual center on simple-to-follow instructions accompanied by clinically relevant comments that keep students motivated and guided them effectively through the dissection process, leaving the faculty more time to teach.

Finally, with the growth of computer graphics, some schools have opted to eliminate anatomy laboratories altogether. However, we contend that virtual learning cannot substitute for comprehensive in-person learning that comes from the sight, touch, and emotion transmitted by well-guided, realistic dissection of human cadavers. To that end, we offer this manual as a resource.

KEYWORDS: Anatomy; dissection manual, health science education 